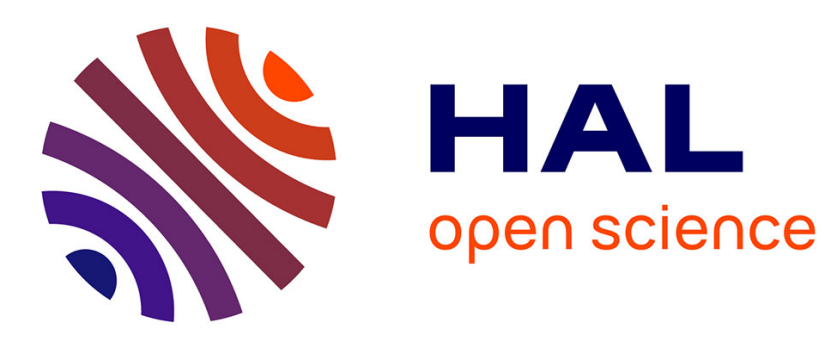

\title{
Spectromètre à double temps-de-vol avec analyse multiparamétrique
}

\author{
C. Perrin, R. Bouchez, P. Quivy, J.C. Gondrand, A. Giorni, J. Pouxe, J.P. \\ Crémet, P. Perrin
}

\section{- To cite this version:}

C. Perrin, R. Bouchez, P. Quivy, J.C. Gondrand, A. Giorni, et al.. Spectromètre à double tempsde-vol avec analyse multiparamétrique. Revue de Physique Appliquée, 1969, 4 (2), pp.111-112. 10.1051/rphysap:0196900402011100 . jpa-00243168

\section{HAL Id: jpa-00243168 https://hal.science/jpa-00243168}

Submitted on 1 Jan 1969

HAL is a multi-disciplinary open access archive for the deposit and dissemination of scientific research documents, whether they are published or not. The documents may come from teaching and research institutions in France or abroad, or from public or private research centers.
L'archive ouverte pluridisciplinaire HAL, est destinée au dépôt et à la diffusion de documents scientifiques de niveau recherche, publiés ou non, émanant des établissements d'enseignement et de recherche français ou étrangers, des laboratoires publics ou privés. 


\title{
SPECTROMÈTRE A DOUBLE TEMPS-DE-VOL AVEG ANALYSE MULTIPARAMÉTRIQUE
}

\author{
C. PERRIN, R. BOUCHEZ, P. QUIVY, J. C. GONDRAND, \\ A. GIORNI, J. POUXE, J. P. GRÉMET et P. PERRIN, \\ Laboratoire de Physique Nucléaire, Institut des Sciences Nucléaires et Centre d'Études Nucléaires de Grenoble.
}

\begin{abstract}
Résumé. - Un ensemble automatique a été réalisé pour l'étude de la réaction à trois nucléons $\mathrm{D}(\mathrm{n}, \mathrm{nnp})$ à $14 \mathrm{MeV}\left(\approx 1\right.$ événement vrai par minute, $\approx 10^{4}$ événements enregistrés), qui permet l'acquisition de sept grandeurs sur bande perforée. L'analyse sur machine IBM 7044 permet ensuite d'obtenir les paramètres de l'interaction nn et np dans l'état ${ }^{1} S_{0}$.
\end{abstract}

Abstract. - The three nucleon reaction $\mathrm{D}(\mathrm{n}, \mathrm{nnp})$ has been studied at $14 \mathrm{MeV}$ using an automatic system for collection of data $\left(\approx 1\right.$ true event by minute, $\approx 10^{4}$ events total). For each event seven parameters were recorded on paper tape. The nn and np interaction parameters were obtained by analysis off-line on an IBM 7044 computer.

I. Introduction. - L'analyse de la réaction à trois nucléons $\mathrm{D}(\mathrm{n}, \mathrm{nnp})$ a été entreprise pour étudier à la fois les interactions ${ }^{1} S_{0}$ singulets nn et np en présence d'un troisième nucléon. L'originalité de l'expérience réside dans : i) le spectromètre à double temps-devol qui donne les distributions biparamétriques $\left(E_{1}, E_{2}\right)$ de l'énergie de chaque neutron final; ii) la mesure, avec le même appareillage, de la distribution biparamétrique $\mathrm{nn}$ ou $\mathrm{np}$; iii) l'acquisition pour chaque événement de sept paramètres : temps-de-vol de chaque neutron (2), identification neutron-gamma pour chaque neutron (2), amplitude de l'impulsion de chaque neutron détecté (2), énergie du proton final (1). Le codage de ces sept paramètres est commandé par une quadruple coïncidence $\alpha-p-n_{1}-n_{2}$; le neutron incident est détecté par sa particule $\alpha$ associée; iv) l'élimination (par machine à calculer) des fausses coïncidences quadruples, dont les paramètres ne sont pas compatibles avec les événements $\alpha-p-n_{1}-n_{2}$, ce qui diminue d'un facteur dix le bruit de fond.

II. Dispositif expérimental. - La salle d'expériences est constituée d'une structure métallique légère. La cible de tritium (réaction $\mathrm{d}(\mathrm{t}, \mathrm{n}) \alpha$ ) se trouve à $2,50 \mathrm{~m}$ du sol et à $4 \mathrm{~m}$ de l'électroaimant de triage du faisceau, ce qui permet de réduire d'un facteur 3,5 le bruit de fond neutron et gamma par rapport à une enceinte de murs épais et avec une cible à $1,50 \mathrm{~m}$ du sol.

Le fonctionnement du générateur de neutrons a été partiellement automatisé en vue de maintenir le faisceau de deutons $(\approx 5 \mu \mathrm{A})$ sur la cible de tritium et de stabiliser l'intensité $\left(\approx 10^{6} \mathrm{n} / \mathrm{s}\right)$ du flux de neutrons. La position du faisceau de deutons est détectée par un système de deux lèvres qui, à l'aide d'un amplifi- cateur différentiel, fournit une tension de sortie proportionnelle au logarithme du rapport des courants recueillis sur les lèvres. Cette tension est réinjectée sur le régulateur comme tension de correction. Le flux de neutrons est maintenu constant par asservissement du courant $\mathrm{d}^{+}$au flux de particules $\alpha$ associées.

III. Détecteurs et identificateurs de particules $\mathbf{n}$ et $\gamma$. - La particule $\alpha$ est détectée par un scintillateur NE $102(4 / 10 \mathrm{~mm})$ associé à un photomultiplicateur XP 1021; le taux de comptage est, au maximum, de $2,5 \times 10^{6} \alpha / \mathrm{s}$. Le proton de cassure du deuton est détecté par un photomultiplicateur 56 AVP 03 à faible bruit et associé à la cible de benzène deutérié $\left(\mathrm{C}_{6} \mathrm{D}_{6}, \mathrm{NE} 230\right.$ : cylindre $\left.\varnothing=5 \mathrm{~cm}, h=12,5 \mathrm{~cm}\right)$. Les neutrons sont détectés dans des réservoirs circulaires $(\varnothing=16 \mathrm{~cm}, e=4 \mathrm{~cm})$ remplis de NE 213 . Ce type de scintillateur organique permet d'identifier les neutrons et les photons $\gamma$, les constantes de temps $\left(\theta_{1}<\theta_{2}\right)$ du scintillateur ainsi que le rapport $A_{1} / A_{2}$ dépendent des particules détectées et du scintillateur [1]. Le seuil de détection des neutrons a pu être abaissé à $600 \mathrm{keV}$, avec une efficacité de détection $(\approx 15 \%)$ pour des énergies de neutron entre 2 et $5 \mathrm{MeV}$.

IV. Mesure des temps-de-vol et électronique rapide. - Le temps-de-vol des deux neutrons $n_{1}$ et $n_{2}$ est mesuré par deux convertisseurs temps-amplitude GTA 02 [2]. Le signal d'ouverture des convertisseurs est fourni par l'alpha associé, en coïncidence, à un proton de cassure de deuton. Cette condition a permis de multiplier, par un facteur cinq, le flux de neutrons incidents sans augmentation du bruit de fond. Un 
événement est repéré par une coïncidence $\left(\alpha p n_{1} n_{2}\right)$. Les plages de coïncidences $\left(\alpha n_{1}\right)$ et $\left(\alpha n_{2}\right)$ sont respectivement de 60 et 120 ns correspondant à la plage d'analyse d'énergie pour les bases de vol 1,20 et 2,40 m.

V. Système d'acquisition des données et électronique logique. - L'ensemble d'acquisition des données comprend sept codeurs (type Wilkinson), un centralisateur de données, une perforatrice de bande pour l'acquisition des résultats, une visualisation numérique des mesures de l'événement, une visualisation (RG 96) sous forme de spectre à une ou deux dimensions par un bloc mémoire $4 \mathrm{k}$. L'ordre d'ouverture aux sept codeurs mis en parallèle est fourni par un dispositif de coïncidence rapide par l'intermédiaire du centralisateur de données, qui permet aussi la séparation en blocs de sept paramètres pour chaque événement ( $\approx 105$ bits $)$.

VI. Traitement des données. - Il est effectué à l'aide de la machine IBM 7044 de la Faculté des Sciences de Grenoble. Les paramètres enregistrés, l'étalonnage des voies et la cinématique des événements $\mathrm{D}(\mathrm{n}, \mathrm{nnp})$ permettent de tracer le spectre d'énergie $T_{\mathrm{p}}$ des protons de cassure, la distribution d'amplitude des impulsions de neutron par rapport à leur énergie $T$ et au signal d'identification $n-\gamma$. On ajuste aussi la fenêtre $\Delta T_{\mathrm{p}}$ pour le proton autour de l'énergie $T_{\mathrm{p}} \approx 4,25 \mathrm{MeV}$. Les événements ( $\mathrm{n}, \mathrm{nnp}$ ) qui répondent aux critères d'analyse sont distribués dans le plan des deux temps-de-vol $t_{1}$ et $t_{2}$ de chaque neutron.

Une simulation de l'expérience a été effectuée par une méthode de Monte-Carlo, pour tenir compte des corrections (résolution angulaire, absorptions...) dues à l'appareillage. La distribution simulée ainsi obtenue est comparée, à l'aide d'une méthode de $\chi^{2}$, à la distribution expérimentale.

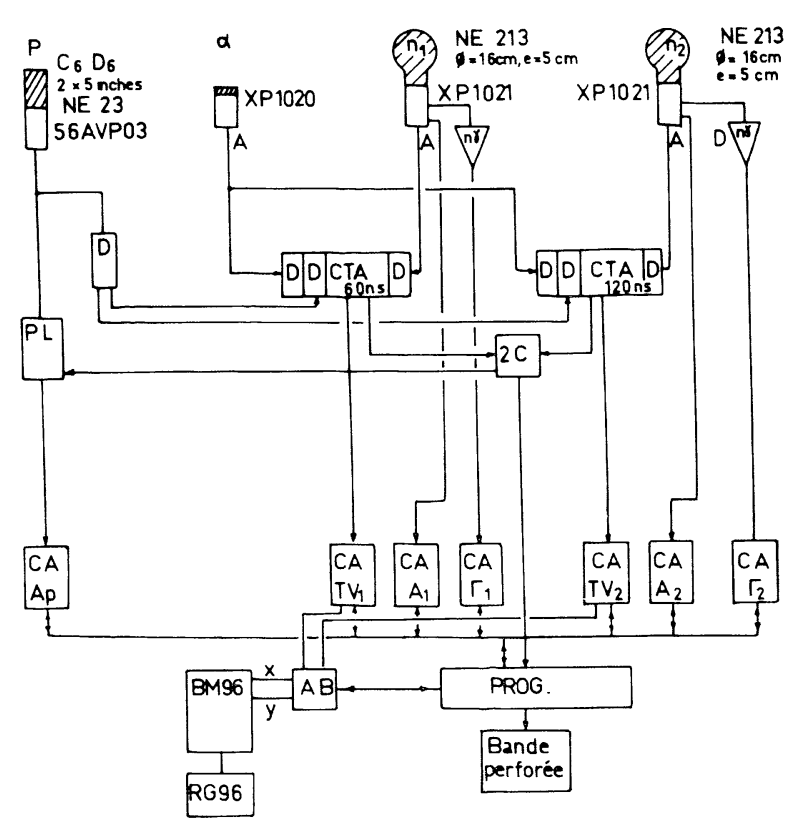

Fig. 1. - Schéma de l'appareillage de l'électronique de détection et de l'électronique logique : $\mathrm{D}(\mathrm{n}, \mathrm{nnp})$ à $14,5 \mathrm{MeV}$; A, B, adresse binaire ; CA, codeurs d'amplitude; Ap, amplitude du proton; $\Gamma_{1} \Gamma_{2}$, identification $\mathrm{n}-\Upsilon$ ?

VII. Résultats préliminaires. - L'expérience nn est terminée. Une première analyse, sans correction de la diffusion multiple, par la méthode des diagrammes de Feynmann, donne pour la longueur de diffusion $a_{\mathrm{nn}} \approx-23 \mathrm{fm}(\approx 15 \%)$ en prenant $r_{\mathrm{nn}}=r_{\mathrm{np}}$ pour la portée de l'interaction $\mathrm{nn}$. L'expérience np est en cours avec le même appareillage que l'expérience nn.

\section{BIBLIOGRAPHIE}

[1] Crémet' (J. P.) et Pouxe (J.), Rapport I.S.N.-68/I 1T, Grenoble, 1968.

[2] VAN ZURK (R.), Rapport C.E.A., R-2405, 1964. 